\title{
Ultrafast control of donor-bound electron spins with single detuned optical pulses
}

\author{
KAI-MEI C. FU ${ }^{1 *}$, SUSAN M. CLARK ${ }^{2}$, CHARLES SANTORI ${ }^{1}$, COLIN R. STANLEY ${ }^{3}$, M. C. HOLLAND ${ }^{3}$ \\ AND YOSHIHISA YAMAMOTO2,4
}

\author{
${ }^{1}$ Information and Quantum Systems Lab, Hewlett-Packard Laboratories, 1501 Page Mill Road, MS1123, Palo Alto, California 94304, USA \\ ${ }^{2}$ Edward L. Ginzton Laboratory, Stanford University, Stanford, California 94305-4088, USA \\ ${ }^{3}$ Department of Electronics and Electrical Engineering, Oakfield Avenue, University of Glasgow, Glasgow, G12 8LT, UK \\ ${ }^{4}$ National Institute of Informatics, 2-1-2 Hitotsubashi, Chiyoda-ku, Tokyo 101-8430, Japan \\ *e-mail: kai-mei.fu@hp.com.
}

The ability to control spins in semiconductors is important in a variety of fields, including spintronics and quantum information processing. Due to the potentially fast dephasing times of spins in the solid state ${ }^{1-3}$, spin control operating on the picosecond timescale, or faster, may be necessary. Such speeds-which are not possible to reach with standard electron spin resonance techniques based on microwave sources-can be attained with broadband optical pulses. One promising ultrafast technique uses single broadband pulses detuned from resonance in a three-level $\Lambda$ system ${ }^{4}$. This technique is robust against optical-pulse imperfections and does not require a fixed optical reference phase. Here we demonstrate, theoretically and experimentally, the principle of coherent manipulation of spins using this approach. Spin rotations with areas exceeding $\pi / 4$ for a single pulse and $\pi / 2$ for two pulses are achieved for donor-bound electrons. This technique might find applications from basic solid-state electron spin resonance spectroscopy to arbitrary single-qubit rotations ${ }^{4,5}$ and bang-bang control ${ }^{6}$ for quantum computation.

Ultrafast optical techniques have previously been used to study semiconductor spins. Faraday rotation and differential transmission can be used to passively study spin dynamics on the picosecond and femtosecond timescale ${ }^{7,8}$. Active techniques so far have used either the optical Stark effect ${ }^{9}$ or Raman transitions through the resonant excitation of an optically active state $e^{2,10-12}$. In contrast to previously demonstrated Raman techniques, in this paper we present a new stimulated Raman transition technique, which is based on a single pulse far detuned from the optical transition. By working off-resonance, decoherence due to real population in the excited state is eliminated without the strict requirement of $2 \pi$ area pulses $^{5}$. We first introduce the theoretical basis for this technique before presenting the experimental results.

We consider the general $\Lambda$-type system with multiple excited states as depicted in Fig. 1. The lower states are denoted $|1\rangle$ and $|2\rangle$ and in the neutral donor system consist of the spin-up and spin-down states of the bound electron. These states are coupled via optical dipole transitions to the $(n-2)$ neutral donor-bound exciton states labelled $|k\rangle$. To see how the spin can be rotated via a single optical pulse, consider the $n$-level Hamiltonian in the rotating frame

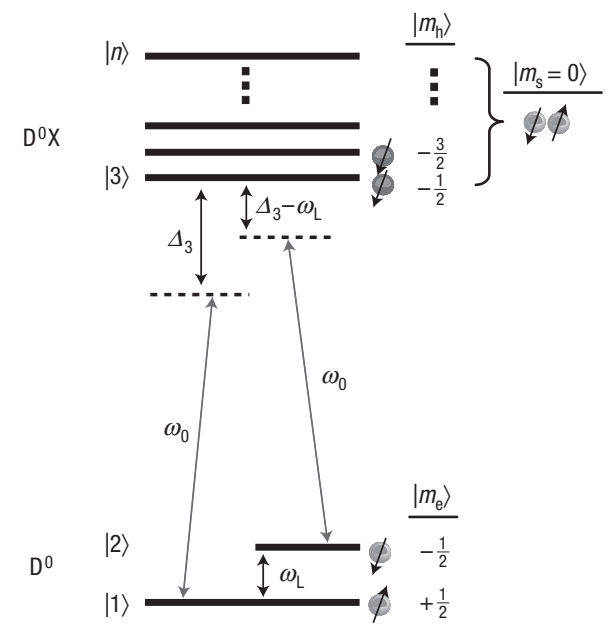

Figure $1 n$-level energy diagram with an applied time-dependent electric field. The two ground levels are split in energy by $\hbar \omega_{L}$. An electric field $E$ with energy $\hbar \omega_{0}$ is applied to the system detuned by the energy $\hbar \Delta_{3}$. Energy levels corresponding to the donor-bound exciton system are denoted to the right of the diagram and are explained further in the Methods section.

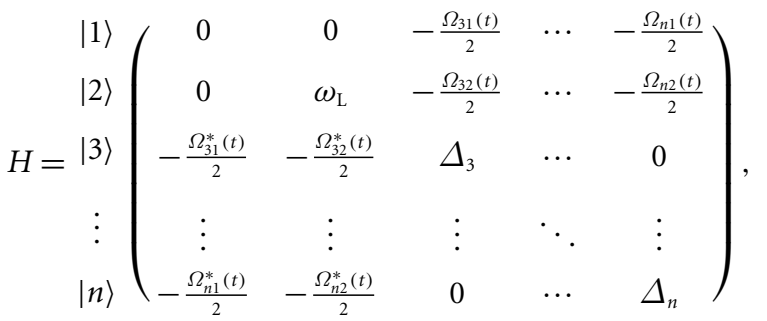

in which $\omega_{\mathrm{L}}$ is the Zeeman splitting of the lower states and $\Delta_{k}$ is the detuning of the applied pulse from the $|1\rangle \leftrightarrow|k\rangle$ transition. The Rabi frequency $\Omega_{k 1}\left(\Omega_{k 2}\right)$ is the product of the dipole matrix element for the $|1\rangle \leftrightarrow|k\rangle(|2\rangle \leftrightarrow|k\rangle)$ transition and the time-dependent electric field amplitude $E$. 

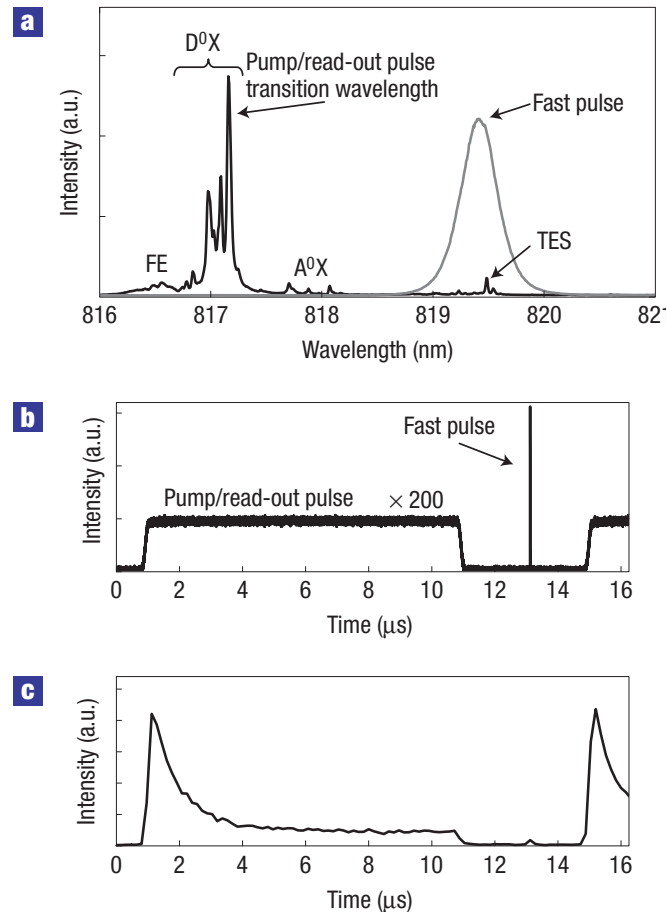

d

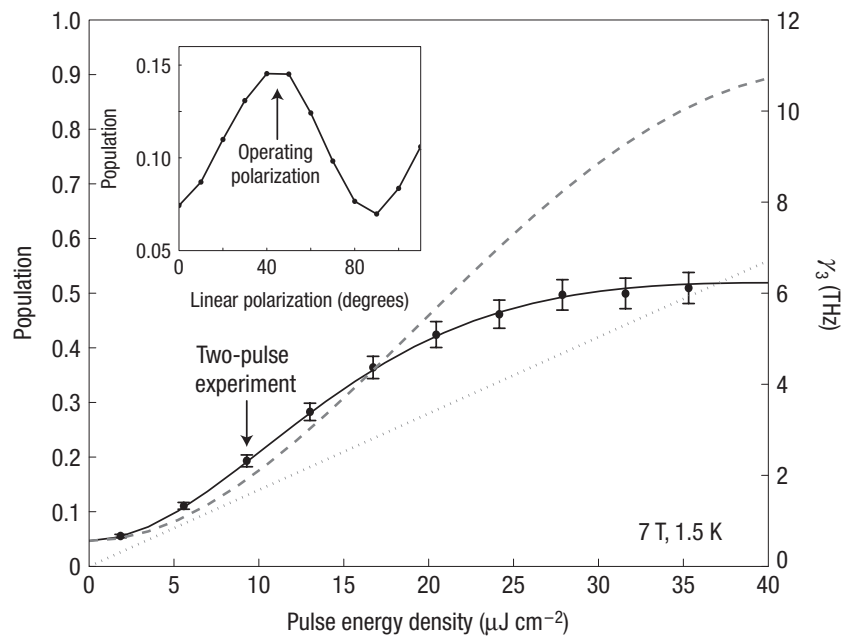

Figure 2 Description of the single-pulse experiment and results. a, Black line: GaAs photoluminescence (PL) spectrum with above-band excitation at $815 \mathrm{~nm}$. Grey line: Fast pulse spectrum tuned $1 \mathrm{THz}$ from the lowest $D^{0} \mathrm{X}$ transition. $\mathbf{b}, \mathrm{A}$ typical laser-pulse sequence recorded on a gigahertz photodiode. $\mathbf{c}, \mathrm{A}$ typical $\mathrm{PL}$ trace collected from the $|3\rangle \rightarrow|1\rangle$ transition as a function of time. d, Symbols: Experimental population in state $|2\rangle$ as a function of pulse energy density. Data were fitted to an exponential to determine the initial PL intensity and thus the initial population in state $|2\rangle$. Error bars are derived from the standard deviation of the fitting coefficients. A saturation at high energies is observed. The arrow marks the pulse energy used for the two-pulse experiment shown in Fig. 3. Dashed line: Three-level simulation assuming a constant

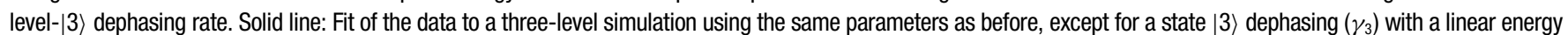
dependency. Dotted line: Peak value of $\gamma_{3}$ used for each pulse energy density in the energy-dependent dephasing model. Inset: Population measured in state $|2\rangle$ as a function of pulse polarization.

This many-level system can be approximated as a two-level spin system by the adiabatic elimination of the upper states, which is valid when the detunings $\Delta_{k}$ are much larger than other rates in the system ${ }^{13}$. The effective two-level Hamiltonian is given by

$$
H_{2}=-\left(\begin{array}{cc}
\frac{\left|\Omega_{1}(t)\right|}{2} & \frac{\Omega_{\mathrm{eff}}(t)}{2} \\
\frac{\Omega_{\mathrm{eff}}(t)^{*}}{2} & \frac{\left|\Omega_{2}(t)\right|}{2}-\omega_{\mathrm{L}}
\end{array}\right),
$$

in which we have defined

$$
\left|\Omega_{1,2}\right|=\frac{1}{2} \sum_{k>2}^{n} \frac{\left|\Omega_{k 1,2}\right|^{2}}{\Delta_{k}}
$$

and an effective Rabi frequency

$$
\Omega_{\mathrm{eff}}=\frac{1}{2} \sum_{k>2}^{n} \frac{\Omega_{k 1} \Omega_{k 2}^{*}}{\Delta_{k}} .
$$

Population is thus coherently transferred from one lower state to the other at the effective Rabi frequency $\Omega_{\text {eff }}(t)$. Note that the optical phase of the pulse is no longer present in this two-level reduction, eliminating the requirement of a fixed optical reference phase as a clock signal. If $\Omega_{\text {eff }}(t) \gg \omega_{\mathrm{L}}$ and $\left|\Omega_{1}\right|=\left|\Omega_{2}\right|$, the rotation axis will be perpendicular to the magnetic field, and full $\pi$ rotations with a single pulse are possible. This condition may often be met by controlling the polarization of the pulse owing to the selection rules for the $|1\rangle \leftrightarrow|k\rangle$ and $|2\rangle \leftrightarrow|k\rangle$ transitions. In material systems in which perpendicular rotations are not possible, large area rotations can still be achieved using multiple pulses by controlling the pulse arrival times over multiple Larmor periods. For a single-pulse rotation, the phase of the rotation is determined by the phase difference between frequency components separated by the Zeeman frequency within the pulse spectrum and thus is determined by the pulse arrival time $e^{4}$. We present the simplified two-level approximation as an intuitive description of the Raman-rotation technique. However, as will be seen below, in a more realistic three-level density-matrix model, which includes excited state relaxation, high-fidelity rotations can still be obtained in a non-adiabatic regime.

One such $\Lambda$-system with multiple excited states that is found in all semiconductors is the neutral donor-bound exciton system. For the experimental demonstration of the Raman technique, we focused on an ensemble measurement of electrons bound to donors in bulk GaAs. At liquid-helium temperatures the donor electron is bound to the donor impurity creating a neutral donor $\left(\mathrm{D}^{0}\right)$. The $\mathrm{D}^{0}$ complex is an attractive potential for excitons (electron-hole pairs) and the resulting neutral donor-bound exciton $\left(\mathrm{D}^{0} \mathrm{X}\right)$ consists of the impurity atom, two bound electrons in a spin-singlet state and a bound hole. The two $\mathrm{D}^{0}$ spin states and multiple $\mathrm{D}^{0} \mathrm{X}$ states are connected by strong, optical transitions ${ }^{14}$, 
and form the lower and excited states of our $n$-level $\Lambda$-type system as shown in Fig. 1.

In the first experiment we demonstrate population transfer between states $|1\rangle$ and $|2\rangle$ with a single pulse in a $7 \mathrm{~T}$ magnetic field. The experiment consisted of three steps: initialization of the spin population into state $|1\rangle$, fast-pulse spin transfer, and population readout of state $|2\rangle$. To initialize the spin state, a continuous-wave field was applied on resonance with the $|2\rangle \leftrightarrow|3\rangle$ transition for $10 \mu \mathrm{s}$. This transition is the brightest transition in the $\mathrm{D}^{0} \mathrm{X}$ spectrum shown in Fig. 2a. At the end of the optical pumping pulse, the state $|1\rangle$ population was 0.94 . After a $2 \mu$ s delay, which was short compared to the longitudinal relaxation between the lower two states $^{15}$, a 2 ps pulse was applied. A typical pulse sequence is shown in Fig. 2b. The pulse was detuned $1 \mathrm{THz}$ below the lowest $\mathrm{D}^{0} \mathrm{X}$ transition. After a second $2 \mu$ s delay the optical pumping pulse was again applied and the population in state $|2\rangle$ was measured by monitoring the photoluminescence (PL) emitted from the $|3\rangle \rightarrow|1\rangle$ transition at the beginning of the pulse. The PL trace as a function of time for a typical case is given in Fig. 2c. The conversion from PL intensity to state $|2\rangle$ population was made by measuring the PL intensity after the system was allowed to return to thermal equilibrium.

As shown above, the relative magnitudes of $\Omega_{1}$ and $\Omega_{2}$ in equation (3), which can be controlled by the pulse polarization, determine the rotation axis and must be equal for rotations about an axis perpendicular to the magnetic field. To obtain the most efficient population transfer possible, the experiment was first carried out at a constant fast-pulse energy for varying pulse polarizations. The polarization dependency is given in Fig. $2 \mathrm{~d}$ inset. Subsequent measurements were made at the peak of this polarization curve. In a system in which Rabi oscillations are observed, the amplitude of these oscillations could be used to determine the rotation axis angle.

In the single-pulse experiment, population transfer was measured as a function of pulse energy by varying the average power of the pulse train. The results are shown in Fig. 2d. At low pulse energies there is a nonlinear increase in population with energy which is characteristic of coherent Raman population transfer. At higher energies, however, population transfer saturates at a value of 0.5 . This saturation was not expected on the basis of a three-level simulation that includes the experimentally reported relaxation rates for the $\mathrm{D}^{0}-\mathrm{D}^{0} \mathrm{X}$ system (see the Methods section). In this simulation, a numerical solution of the master equation,

$$
\dot{\rho}=-i[H, \rho]+\mathcal{L}(\rho)
$$

is obtained, where $\rho$ is the three-level density matrix, $H$ is the Hamiltonian given in equation $(6)$ and $\mathcal{L}(\rho)$ is the relaxation super-operator given in equation (7). In the full density-matrix formalism we find that, although the evolution is not adiabatic and virtual excitation of the excited state occurs, high-fidelity rotations are still possible. Applying a 2 ps full-width at half-maximum hyperbolic secant pulse with a detuning $\Delta_{3}=1 \mathrm{THz}$ and using the definition of fidelity $F=\langle\psi|\rho| \psi\rangle$, where $|\psi\rangle$ is the desired quantum state, we found that $\pi$-rotations should have been possible with a 0.97 fidelity and $\pi / 2$ rotations should have been possible with $>0.99$ fidelity for $\left|\Omega_{1}\right|=\left|\Omega_{2}\right|$. However, a good fit to the experimental data can be obtained using a three-level model that includes a dephasing rate of the excited state $\left(\gamma_{3}\right)$ that is linearly dependent on the pulse energy. Although Rabi oscillations were not observed in this first experiment, the non-linear increase in population transfer suggests that small coherent rotations are possible at low powers.

To measure the coherence of the small-angle rotations, a second experiment with two fast pulses was carried out. In the
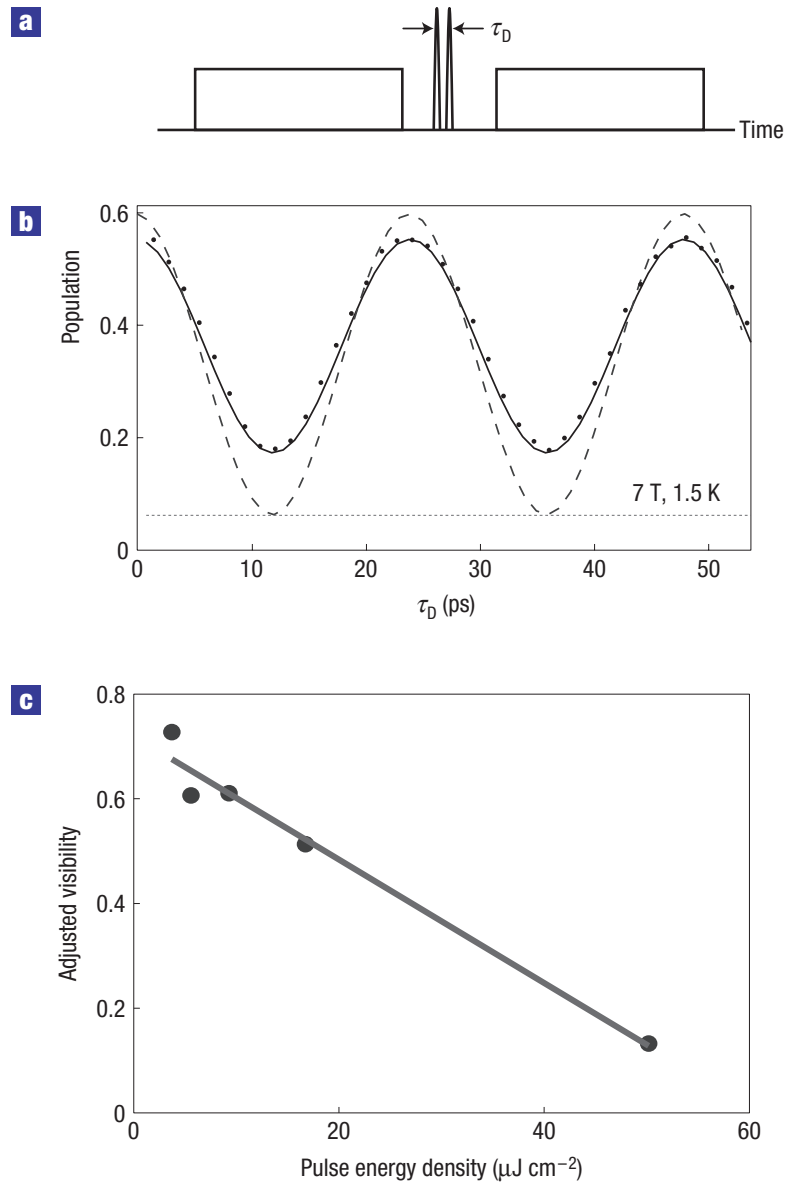

Figure 3 Description of the double-pulse experiment and results. a, Schematic diagram of the double-pulse-experiment pulse sequence. $\mathbf{b}$, Symbols: Population in state $|2\rangle$ after the second pulse as a function of $\tau_{\mathrm{D}}$. Solid black line: The simulation result that uses a level $|3\rangle$ dephasing of $1.6 \mathrm{THz}$ consistent with the energy-dependent dephasing model. Dashed line: Three-level simulation with a $10 \mathrm{GHz}$ level $|3\rangle$ dephasing rate. Dotted line: Residual population remaining in state |2〉 after the optical pumping pulse. c, Pulse-energy dependency of the two-pulse visibility. The solid line is a linear fit. The visibility is defined as $\left(I_{\max }-I_{\min }\right) /\left(I_{\max }+I_{\min }\right)$, in which $I_{\max }\left(I_{\min }\right)$ is the maximum (minimum) of the two-pulse visibility curve after subtracting the intensity observed after optical pumping.

double-pulse experiment the single pulse was split into two pulses with a variable delay $\tau_{\mathrm{D}}$ (Fig. $3 \mathrm{a}$ ). As the delay between the two pulses increases, the final population in state $|2\rangle$ oscillates with a period equal to the Larmor period $\tau_{\mathrm{L}}$. In Fig. $3 \mathrm{~b}$ we plot the population in state $|2\rangle$ after two pulses of energy $10 \mu \mathrm{J} \mathrm{cm}^{-2}$ were applied, as a function of $\tau_{\mathrm{D}}$. An oscillation at the Larmor frequency is clearly observed, verifying coherent population transfer as well as rotation axis control. The observed $42 \mathrm{GHz}$ oscillation at $7 \mathrm{~T}$ corresponds to a $\mathrm{D}^{0}$ electron $g$-factor of $g_{\mathrm{e}}=-0.42$, which is consistent with previous measurements ${ }^{16}$. In contrast to the ideal case, the population in state $|2\rangle$ never reaches the optically pumped value and perfect destructive interference does not occur. The finite population left in state $|2\rangle$ indicates that, in addition to coherent population transfer, incoherent population transfer occurs. The simultaneous fit of the single- (Fig. 2d) and double-pulse data (Fig. 3b) to the energy-dependent dephasing model indicates a 


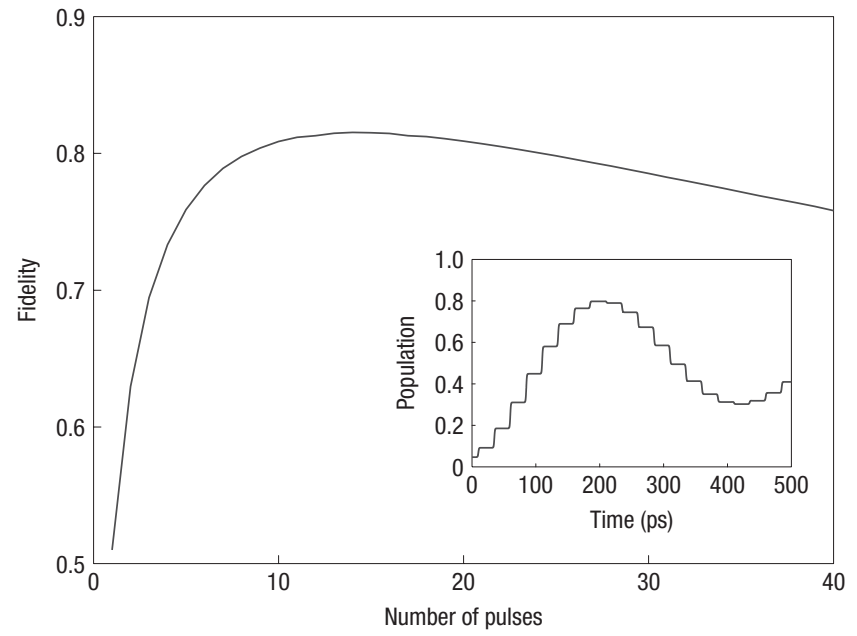

Figure 4 Theoretical calculation of the fidelity of $\pi$-pulses versus number of pulses applied in phase. Inset: Population in state $|2\rangle$ versus time as 2 ps pulses are applied in phase.

single-pulse rotation of 0.9 radians and a double-pulse rotation of 1.8 radians, with fidelities 0.85 and 0.78 respectively. The two-pulse experiment was carried out at several powers, with a linear decrease in the visibility observed with increasing power as shown in Fig. 3c.

The saturation of the population transfer in the first experiment and the absence of perfect destructive interference in the second experiment indicate that some fast dephasing mechanism is occurring in the $\mathrm{D}^{0}-\mathrm{D}^{0} \mathrm{X}$ system. This behaviour is not expected from the known parameters in the $\mathrm{D}^{0} \mathrm{X}$ system in the low-excitation limit $^{1}$. However, the single-impurity three-level model may not be sufficient to describe the experimental many-exciton system. We note that, in addition to exciting an appreciable virtual $\mathrm{D}^{0} \mathrm{X}$ population during the applied pulse, virtual free excitons are also excited. As shown in Fig. 2a, the $\mathrm{D}^{0} \mathrm{X}$ transitions lie right on the tail of the free-exciton transitions. Unlike the $\mathrm{D}^{0} \mathrm{X}$ transitions, the free-exciton transition is fairly broad $(\mathrm{THz})$ and thus additional real excitation due to the picosecond pulse is likely. We expect the incoherent free-exciton excitation to be linear in pulse energy until the free-exciton transition has saturated. Once free excitons are excited, exciton-exciton interactions and exciton-electron interactions ${ }^{17,18}$ could be the source of the observed fast dephasing and a many-particle model may be necessary to explain the experiment result. Dephasing due to multi-exciton interactions should be less of a factor in deeper-impurity systems ${ }^{19}$ or charged III-V quantum-dot systems ${ }^{2,10,11}$, which have much larger exciton binding energies than the GaAs $\mathrm{D}^{0} \mathrm{X}$ system. In these systems it may be possible to obtain single-pulse large-area spin rotations, which would be a valuable tool for spin-based quantum information processing.

Even with the modest population transfer possible in our bulk experimental system, simulations indicate that $\pi$-pulses are still possible if several low-energy pulses are applied to the system in phase. Again, using the three-level model that assumes a level $|3\rangle$ dephasing rate $\left(\gamma_{3}\right)$ with a linear power dependency, we find that a $\pi$-rotation is possible with 0.80 fidelity in as few as eight pulses (200 ps) with energy densities of $5 \mu \mathrm{J} \mathrm{cm}^{-2}$ each. As visible in the Fig. 4 inset, applying small-angle pulses in succession results in a discrete Rabi oscillation curve. Figure 4 shows the fidelity of a $\pi$-rotation as a function of the number of pulses applied in phase. We might expect the fidelity to increase to unity as more and more weaker pulses are used to carry out the rotation. However, the finite decoherence time of $1 \mathrm{~ns}$ limits the total number of pulses that can be used. $1 \mathrm{~ns}$ is the experimental inhomogeneous dephasing time $T_{2}^{*}$ in the system ${ }^{1}$, and the decoherence time could be much longer for a single-spin system. While the multiple-pulse technique is significantly slower than the single-pulse technique, it may prove valuable in systems in which large-area rotations are desired yet low powers are necessary. Such large-area rotations are necessary for single gates in quantum computation and can also aid in the suppression of decoherence ${ }^{6}$. However, for general electron spin resonance techniques, such as spin echo, small-area pulses are all that is necessary for determining the fundamental homogeneous decoherence time $T_{2}$ (ref. 20) in a material. Thus this Raman fast-pulse technique as experimentally demonstrated can immediately be applied for these studies.

Note added in proof. After the submission of this work we became aware of similar work using detuned optical pulses to control the electron spin in a quantum $\operatorname{dot}^{21}$.

\section{METHODS}

\section{THE $D^{0} X$ SYSTEM}

The sample studied consisted of a $10 \mu \mathrm{m}$ GaAs layer with a donor density of $5 \times 10^{13} \mathrm{~cm}^{-3}$ on a $4 \mu \mathrm{m} \mathrm{Al}_{0.3} \mathrm{Ga}_{0.7}$ As layer grown by molecular-beam epitaxy on a GaAs substrate. The sample was mounted strain free in a magnetic cryostat in a liquid-helium bath. The magnetic field was parallel to the $\langle 110\rangle$ crystallographic axis. The magnetic field was perpendicular to the excitation and collection paths. The signal was collected from a $20 \mu \mathrm{m}$ spot with an estimated $10^{5}$ donors contributing to the signal.

In the applied magnetic field, the neutral donor electron splits into two levels denoted by the magnetic quantum number $m_{\mathrm{e}}= \pm(1 / 2)$ as shown in Fig. 1 . The bound-electron $g$-factor $g_{\mathrm{e}}$ is -0.40 to -0.46 depending on the strength of the magnetic field and the crystal orientation with respect to the magnetic field ${ }^{16}$. The excited-state $\mathrm{D}^{0} \mathrm{X}$ energy-level structure is much more complex, and although it is not fully understood a detailed study has been carried out ${ }^{16}$. The two electrons in the complex form a spin singlet denoted in Fig. 1 by $\left|m_{s}=0\right\rangle$. The energy of the $\mathrm{D}^{0} \mathrm{X}$ state is thus determined by the spin of the bound hole $m_{\mathrm{h}}= \pm(1 / 2), \pm(3 / 2)$ as well as the hole's effective mass orbital angular momentum $L$. In our system we have identified the ground and first excited states of the $\mathrm{D}^{0} \mathrm{X}$ complex as the $\left|L=1, m_{\mathrm{h}}=-(1 / 2)\right\rangle$ and the $\left|L=0, m_{\mathrm{h}}=-(3 / 2)\right\rangle$ states respectively.

A GaAs photoluminescence spectrum with above-band excitation at $815 \mathrm{~nm}$ can be seen in Fig. 2a (black curve). Only the horizontal polarization is collected to resolve more of the $\mathrm{D}^{0} \mathrm{X}$ transitions, and $\mathrm{D}^{0} \mathrm{X}$ linewidths are instrument resolution limited. Also observed in the spectrum are the free-exciton (FE) transitions, the acceptor bound-exciton $\left(\mathrm{A}^{0} \mathrm{X}\right)$ transitions and the $\mathrm{D}^{0} \mathrm{X}$ two-electron satellite (TES) transitions. TES transitions occur when the $\mathrm{D}^{0} \mathrm{X}$ relaxes into a $\mathrm{D}^{0}$ excited state.

High-resolution photoluminescence excitation spectroscopy as well as the location of the two-electron satellite lines indicate that the primary donor in our sample is silicon with a much weaker $(<10 \times)$ concentration of sulphur. Photoluminescence excitation spectroscopy on the sample shows that the inhomogeneous linewidth is $<10 \mathrm{GHz}$ on the broadest lines ${ }^{1}$. This linewidth is much narrower than the $1 \mathrm{THz}$ detuning, and thus should not affect the fidelity of the pulse rotations.

\section{ADDITIONAL INFORMATION ON THE EXPERIMENTAL PULSE SEQUENCE}

A typical fast-pulse sequence detected on a gigahertz photodiode is shown in Fig. 2b, in which the pump/read-out pulse intensity is 200 times the intensity used in the experiment. The optical pumping laser was a Coherent Ti:sapphire 899-29 continuous-wave laser modulated by an AOM and polarized parallel to the magnetic field. Depending on the experiment, the pump power ranged from 5 to $15 \mu \mathrm{W}$ and the spot size was approximately $120 \mu \mathrm{m}$. The fast pulse was provided by a picosecond mode-locked laser with a repetition rate of $80 \mathrm{MHz}$. Single pulses were picked by an EOM every 10-15 $\mu$ s depending on the particular experiment. The EOM extinction ratio ranged from 60 to 100 and an additional $2 \mu$ s extinction envelope was provided by an AOM (extinction ratio 
of 1,000$)$. The fast-pulse polarization was $45^{\circ}$ from the magnetic field axis. The fast-pulse laser spot size was $80 \mu \mathrm{m}$.

\section{THREE-LEVEL THEORETICAL MODEL}

The data in Figs $2 \mathrm{~d}$ and $3 \mathrm{~b}$ are fitted to a three-level density-matrix model that solves the master equation given in equation (5). The three-level Hamiltonian in the rotating frame is given by

$$
H_{3}=\left(\begin{array}{ccc}
0 & 0 & -\frac{\Omega_{31}(t)}{2} \\
0 & \omega_{\mathrm{L}} & -\frac{\Omega_{32}(t)}{2} \\
-\frac{\Omega_{31}^{*}(t)}{2} & -\frac{\Omega_{32}^{*}(t)}{2} & \Delta
\end{array}\right),
$$

and the relaxation operator $\mathcal{L}(\rho)$ is given by

$$
\mathcal{L}(\rho)=\left(\begin{array}{r}
-\Gamma_{12} \rho_{11}+\Gamma_{21} \rho_{22}+\Gamma_{3} \rho_{33} \\
-\left(\frac{\Gamma_{12}+\Gamma_{21}}{2}+\gamma_{2}\right) \rho_{21} \\
-\left(\frac{\Gamma_{12}+2 \Gamma_{3}}{2}+\gamma_{3}(t)\right) \rho_{31} \\
\quad-\left(\frac{\Gamma_{12}+\Gamma_{21}}{2}+\gamma_{2}\right) \rho_{12} \\
\Gamma_{12} \rho_{11}-\Gamma_{21} \rho_{22}+\Gamma_{3} \rho_{33} \\
-\left(\frac{\Gamma_{21}+2 \Gamma_{3}}{2}+\gamma_{3}(t)\right) \rho_{32} \\
-\left(\frac{\Gamma_{12}+2 \Gamma_{3}}{2}+\gamma_{3}(t)\right) \rho_{13} \\
-\left(\frac{\Gamma_{21}+2 \Gamma_{3}}{2}+\gamma_{3}(t)\right) \rho_{23} \\
-\left(2 \Gamma_{3}\right) \rho_{33}
\end{array}\right),
$$

in which $\Gamma_{12}\left(\Gamma_{21}=\Gamma_{12} \mathrm{e}^{\left(E_{12} / k T\right)}\right)$ is the longitudinal relaxation rate from $|1\rangle \rightarrow|2\rangle(|2\rangle \rightarrow|1\rangle), 2 \Gamma_{3}$ is the total radiative relaxation from $|3\rangle, \gamma_{2}$ is the transverse relaxation rate between $|1\rangle$ and $|2\rangle$ and $\gamma_{3}(t)$ is the level $|3\rangle$ dephasing. For electrons bound to neutral donors in GaAs, the relevant relaxation parameters are the excited-state radiative relaxation rate $2 \Gamma_{3}=(1 \mathrm{~ns})^{-1}$ (ref. 14), the lower-state decoherence time $T_{2}=1 \mathrm{~ns}$ (ref. 1) and a Zeeman splitting of $\omega_{\mathrm{L}}=42 \mathrm{GHz}$ in a $7 \mathrm{~T}$ field. In the constant level|3〉 dephasing model, $\gamma_{3}(t)=10 \mathrm{GHz}$ (refs 1,16). Three-level simulations show that even in this non-adiabatic regime $\mathrm{D}^{0} \mathrm{X}$ population is only virtually excited during the pulse duration. Pulse fidelities are high as long as all dephasing mechanisms in the system are slow compared with the pulse time. In the intensity-dependent model the peak value of $\gamma_{3}(t)$ is given in Fig. 2d. The model fits the data if pulse energies are 0.8 times the energies in the experiment. This discrepancy could be explained by our incomplete knowledge of the $\mathrm{D}^{0} \mathrm{X}$ dipole matrix elements. The model includes only one excited state, with a relaxation rate equal to the experimental total relaxation rate for the many-level $\mathrm{D}^{0} \mathrm{X}$ state at zero magnetic field. In reality, our fast pulse is interacting with many levels in a $7 \mathrm{~T}$ field.
Received 11 December 2007; accepted 7 July 2008; published 10 August 2008.

References

1. Fu, K. M. C., Santori, C., Stanley, C., Holland, M. C. \& Yamamoto, Y. Coherent population trapping of electron spins in a high-purity n-type GaAs semiconductor. Phys. Rev. Lett. 95, 187405 (2005)

2. Gurudev Dutt, M. V. et al. Ultrafast optical control of electron spin coherence in charged GaAs quantum dots. Phys. Rev. B 74, 125306 (2006).

3. Titkov, A. N., Safarov, V. I. \& Lampel, G. in Physics of Semiconductors (ed. Wilson, B. L. H.) 1031-1034 (Institute of Physics, Bristol, 1978).

4. Clark, S. M., Fu, K.-M. C., Ladd, T. D. \& Yamamoto, Y. Quantum computers based on electron spins controlled by ultrafast off-resonant single optical pulses. Phys. Rev. Lett. 99, 40501 (2007).

5. Economou, S. E., Sham, L. J., Wu, Y. \& Steel, D. G. Proposal for optical u(1) rotations of electron spin trapped in a quantum dot. Phys. Rev. B 74, 205415 (2006).

6. Viola, L. \& Lloyd, S. Dynamical suppression of decoherence in two-state quantum systems. Phys. Rev. A 58, 2733-2744 (1998)

7. Crooker, S., Awschalom, D., Baumberg, J. J., Flack, F. \& Samarth, N. Optical spin resonance and transverse spin relaxation in magnetic semiconductor quantum wells. Phys. Rev. B 56 7574-7588 (1997).

8. Ferrio, K. B. \& Steel, D. G. Raman quantum beats of interacting excitons. Phys. Rev. Lett. 80 786-789 (1998)

9. Gupta, J. A., Knobel, R., Samarth, N. \& Awschalom, D. D. Ultrafast manipulation of electron spin coherence. Science 292, 2458-2461 (2001).

10. Greilich, A. et al. Mode locking of electron spin coherence in singly charged quantum dots. Science 313, 341-345 (2006)

11. Wu, Y. et al. Selective optical control of electron spin coherence in single charged $\mathrm{GaAs}-\mathrm{Al}_{0.3} \mathrm{Ga}_{0.7} \mathrm{As}$ quantum dots. Phys. Rev. Lett. 99, 97402 (2007).

12. Shen, Y., Goebel, A. M. \& Wang, H. Control of quantum beats from electron spin coherence in semiconductor quantum wells. Phys. Rev. B 75, 045341 (2007).

13. Harris, S. E. Refractive-index control with strong fields. Opt. Lett. 19, 2018-2020 (1994).

14. Finkman, E., Sturge, M. D. \& Bhat, R. Oscillator strength, lifetime, and degeneracy of resonantly excited bound excitons in GaAs. J. Lumin. 35, 235-238 (1986).

15. Fu, K.-M. C. et al. Millisecond spin-flip times of donor-bound electrons in GaAs. Phys. Rev. B 74 121304 (2006).

16. Karasyuk, V. A. et al. Fourier-transform magnetophotoluminescence spectroscopy of donor-bound excitons in GaAs. Phys. Rev. B 49, 16381-16397 (1994)

17. Piermarocchi, C., Chen, P., Sham, L. J. \& Steel, D. G. Optical RKKY interaction between charged semiconductor quantum dots. Phys. Rev. Lett. 89, 16702 (2002).

18. Paget, D. Optical detection of NMR in high-purity GaAs under optical pumping: Efficient spin-exchange averaging between electronic states. Phys. Rev. B 24, 3776-3793 (1981).

19. Strauf, S. et al. Quantum optical studies on individual acceptor bound excitons in a semiconductor Phys. Rev. Lett. 89, 177403 (2002)

20. Rosatzin, M., Suter, D. \& Mlynek, J. Light-shift-induced spin echoes in a $j=1 / 2$ atomic ground state. Phys. Rev. A 42, 1839-1841 (1990).

21. Berezovsky, J., Mikkelson, M. H., Stoltz, N. G., Coldren, L. A. \& Awschalom, D. D. Picosecond coherent manipulation of a single electron spin in a quantum dot. Science 320, 349-352 (2008).

\section{Acknowledgements}

The authors thank S. Harris and J. Pelc for discussions. S.M.C. was partially supported by the HP Fellowship Program through CIS. This work was financially supported by the MURI Center for Photonic Quantum Information Systems (ARO/ARDA Program DAAD 19-03-1-0199), the JST/SORST Program for the Research of Quantum Information Systems for Which Light is Used, and the University of Tokyo (CINQIE) Special Coordination Funds for Promoting Science and Technology, the "Qubus quantum computer program" MEXT and NICT program on Quantum Repeaters.

\section{Author contributions}

C.R.S. and M.C.H. provided the sample. K-M.C.F and S.M.C. designed, built and carried out the experiment. K-M.C.F. analysed and modelled the experimental data and did the majority of the writing, with significant contributions from S.M.C. S.M.C. contributed the theoretical multiple-small-angle-pulse model. C.S. and Y.Y. contributed throughout the experimental process from the conception to the writing through useful suggestions, comments and discussions.

\section{Author information}

Reprints and permission information is available online at http://npg.nature.com/reprintsandpermissions Correspondence and requests for materials should be addressed to K-M.C.F. 